\title{
An anatomical and clinical review of cricopharyngeal achalasia in the dog
}

\author{
R C Elliott ${ }^{\mathrm{a}}$
}

\begin{abstract}
Cricopharyngeal achalasia is a rare cause of dysphagia in the dog. However it must be differentiated from other causes of dysphagia as it is treatable with surgery. It is a disruption of the cricopharyngeal phase of the oropharyngeal phase of deglutition. There appears to be an incoordination in the swallowing process between the relaxation of the rostral, middle pharyngeal muscles and the caudal pharyngeal muscles. It is seen as a primary condition in young animals presenting soon after weaning onto solid food. The dogs appear clinically healthy unless there is secondary aspiration pneumonia or emaciation. These dogs may present as respiratory emergencies and require intensive support and treatment prior to corrective surgery. The diagnosis is made on videofluoroscopy. The condition carries a good prognosis for cure with surgical myectomy of the cricopharyngeal muscle and the thyropharyngeal muscle, which make up the upper oesophageal sphincter. Temporary relief prior to surgery can be achieved by injection of the cricopharyngeal muscle with botulism toxin. Surgical treatment for dysphagia secondary to an underlying neurological, neuromuscular or pharyngeal weakness carries a guarded prognosis and will make aspiration pneumonia worse.
\end{abstract}

Keywords: achalasia, aspiration pneumonia, botulism, cricopharyngeal myectomy, dysphagia, myotomy, toxin.

Elliott R C An anatomical and clinical review of cricopharyngeal achalasia in the dog Journal of the South African Veterinary Association (2010) 81(2): 75-79 (En.). Bryanston Veterinary Hospital, PO Box 67092, Bryanston, 2021 South Africa.

\section{INTRODUCTION}

Cricopharyngeal achalasia (CA) is a rare disorder of dysphagia of the upper oesophageal sphincter (UES) that is usually congenital ${ }^{9}$. This sphincter prevents the swallowing of air during respiration and escape of contents of the proximal oesophagus into the pharynx where they can be aspirated ${ }^{13,16,19}$. It is vital to differentiate it from other forms of dysphagia as it is treatable with surgery ${ }^{1,4}$. The underlying mechanism is unknown but appears to be linked to incoordination in the swallowing reflex. This is either due to a failure of the UES to open during the swallowing phase, or due to an incoordinated opening in relation to the rest of the pharyngeal muscles. Both will be encompassed in the term cricopharyngeal achalasia in this article $e^{4,5,7}$. The clinical signs can be reproduced by transecting the pharyngeal branch of the vagus nerve. There are no consistent histopathological changes in the affected muscle fibres ${ }^{4,16}$. The clinical disease of CA is a rare presentation to the veterinarian. The veterinarian has to have a high index of suspicion to assist in making the diagnosis. It is hoped that this clinical

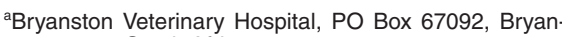
ston, 2021 South Africa.

E-mail: rosselliott_2@ hotmail.com

Received: February 2010. Accepted: May 2010. review will increase the awareness of this rare but easily treatable disease in the small animal patient, giving affected individuals a good chance of a relatively good life.

Cricopharyngeal achalasia is seen in humans as a rare clinical disease. It is classified as primary without a concurrent underlying disease or secondary when it is caused by an underlying disease. It is seen in adults usually as a result of neurological disease. The primary form is seen in infants as a rare cause of dysphagia. It is often not diagnosed until late in infancy due to rare distribution of the disease. It can be associated with other congenital defects of the upper gastrointestinal system ${ }^{2,6,10}$.

In humans the cricopharyngeal muscle has been shown to be made up of $72 \%$ type I myosin heavy chain fibres. It was, however, found that there is no statistical difference in the isoform and type of myosin heavy chain fibres between humans with CA and normal patients ${ }^{2}$.

\section{NORMAL DEGLUTITION PHASES}

During mastication there are 3 phases of deglutition - the oropharyngeal phase, the oesophageal phase and the gastrooesophageal phase $\mathrm{e}^{16,19}$. The oropharyngeal phase is divided into the following phases. The oral phase, the pharyngeal phase, the cricopharyngeal phase and the oesophageal phase. These phases form a complex reflex action that coordinates many structures including the cranial nerves, swallowing centre in the reticular formation, muscles of mastication, tongue, soft palate, pharynx, larynx and oesophagus $^{9,16,19}$.

The oral phase starts when mastication is complete and the food is moved to the base of the tongue. This phase is volun$\operatorname{tary}^{16,19}$.

The pharyngeal phase is initiated by sensory nerves in the oropharynx. The goal of the pharyngeal phase is to transport the bolus of food from the oropharynx to the UES through the pharynx while preventing aspiration ${ }^{9,16,19}$. This is characterised by a wave-like relaxation to contraction of the rostral, middle and caudal pharyngeal muscles in a sequential fashion thus moving the bolus of food. During this contraction the soft palate is elevated, the palatopharyngeal folds move inward, vocal folds are moved together and the larynx is elevated against the epiglot$\operatorname{tis}^{16,19}$. The pharyngeal recesses will further protect the airway as they divert fluid and food material away from the airway $^{3,16,19}$.

As the bolus reaches the caudal pharyngeal muscles it enters the cricopharyngeal phase. The cricopharyngeal and the thyropharyngeal muscles make up the UES $^{16,19}$. This phase is characterised by relaxation of these muscles and food entering into the oesophagus. This relaxation, which occurs in synchrony with the contraction of the cranial and middle pharyngeal muscles, allows the entire bolus to enter the oesophagus, after which contraction of the UES prevents reflux ${ }^{3,16,19}$.

The oesophageal phase transports the bolus from the oesophagus to the gastrooesophageal sphincter ${ }^{16,19}$. As the bolus enters the oesophagus, it stimulates a peristaltic wave, sweeping the bolus to the gastro-oesophageal junction. This is known as primary peristalsis. If there is a retained bolus, a second peristaltic wave is stimulated to sweep the remaining bolus to the gastro-oesophageal junction. Secondary peristalsis is initiated by mechanical stimulation of tactile stretch of the retained bolus on the wall of the 
oesophagus. This may occur up to 2-3 times in a normal animal ${ }^{16,19}$.

The gastro-oesophageal phase is the slow transport of food through the gastro-oesophgeal sphincter to the lower oesophageal sphincter into the stom$\operatorname{ach}^{16,19}$.

\section{NERVE CONTROL OF DEGLUTITION}

Cranial nerve IX, the glossopharyngeal nerve, provides sensory innervations to the pharynx and a portion of the tongue. Its motor fibres run with the vagus and innervate most of the constrictors and dilators of the pharyn $x^{3,16,19}$. Cranial nerve $X$, the vagus nerve, is most intimately involved in swallowing. As described above it provides both visceral efferent and branches to the pharynx forming the pharyngo-oesophageal nerve ${ }^{19}$. This provides the motor innervations to the caudal pharyngeal muscles, the laryngeal muscles and the cervical oesophagus. The trigeminal nerve $\mathrm{CN} \mathrm{V}$ consists of sensory fibres from the oral cavity and motor fibres to the muscles of mastication and the mylohyoideus and the muscles of the soft palate ${ }^{3,16,19}$. The hypoglossal nerve CN XII supplies the motor fibres to the extrinsic and intrinsic muscles of the tongue. The facial nerve CN VII contains sensory fibres from receptors in the soft palate and nasopharynx. CN VII supplies motor fibres to the stylohyoid and jugulohyoid muscles ${ }^{3,16,19}$. These nerves all control the complex process of deglutition including the function of the lips, masticatory muscles, tongue, soft palate, pharynx and UES. Central disorders are rare causes of deglutition but diseases involving the nuclei of these nerves may result in dysphagia ${ }^{16,19}$.

\section{ROLE OF THE MUSCLES OF THE PHARYNX}

The muscles of the pharynx that play a central role are as follows and they are all innervated by CN IX and CN X. The rostral pharyngeal constrictors tend to be poorly developed and their fibres tend to intermingle with each other. They are made up of 2 parts, the pterygopharyngeal muscle which arises from the Hamulus pterygoideus and inserts on the median fibrous raphe $e^{3}$ and the palatopharyngeal muscle which arises from the soft palate, both inserting on the median fibrous raphe ${ }^{3}$. The middle pharyngeal constrictor, the hyopharyngeal muscle, originates from the lateral surface of the thyrohyoid and ceratohyoid bones and inserts on the median fibrous raphe. The fibres of insertion from this muscle can overlie the fibres from the thyopharyngeal muscle ${ }^{3}$. The caudal pharyngeal constrictors, the cricopharyngeal and thyropharyngeal muscles, form the upper oesophageal sphincter. The thyropharyngeal muscle originates from the lamina of the thyroid cartilage of the larynx and inserts on the median fibrous raphe dorsal to the phar$y n x^{3}$. The cricopharyngeal muscle originates from the cricoid cartilage of the larynx and inserts on the median fibrous raphe caudal to the thyropharyngeal muscle $^{3}$.

Cricopharyngeal achalasia is characterised by inadequate relaxation of the cricopharyngeal muscle in coordination with the other pharyngeal muscles during swallowing. The bolus of food remains in the pharynx after repeated swallowing attempts $^{1,5,7,9}$. Some of the bolus may pass into the oesophagus and then moves normally to the stomach. Some of the food may be aspirated and lead to recurrent or chronic aspiration pneumonia $^{4,5,7}$. Some of the food may be regurgitated.

\section{DIAGNOSIS}

In making the diagnosis the clinician has to be sure there is no underlying disease. If surgical correction is attempted without ruling out other causes of dysphagia, this can result in worsening of the condition. The patient will be put through an invasive surgical procedure with very little clinical benefit. A complete list of differential diagnoses is beyond the scope of this review. There is, however, a list of the common differentials that need to be ruled out

\section{Differential diagnosis}

Oral dysphagia

Dental disease

Oral masses/foreign bodies

Cleft palate

Oral trauma

Disease of the temporamandibular joint

Pharyngeal dysphagia

Cricopharyngeal achalasia

Pharyngeal inflammation

Obstruction/foreign body/neoplasia

Trauma/stick injury

Nasopharyngeal dysgenesis

Pharyngeal mucocele

Oesophageal dysphagia

Oesophagitis/Stricture

Myasthenia gravis

Spirocercosis

Foreign body

Vascular ring anomalies

Hyperthyroidism

Hiatal hernia

Neuro-muscular

Acute polyradiculitis

Tick paralysis

Botulism

Dystrophin-deficient muscular dystrophy
Neurological

Inflammatory CNS disease

Hydrocephalus

Rabies

\section{SIGNALMENT}

The condition is relatively rare, but seems to be more common in Springer and Cocker spaniels. The condition is commonly seen in animals under a year of age but is rarely diagnosed in older animals $^{1,4,7}$.

\section{Physical examination}

Dogs appear normal until they are weaned onto solid food, when they make repeated attempts to swallow, with gagging, retching and expulsion of saliva-covered bolus ${ }^{11,17}$. Most patients are ravenous but show weight loss and dysphagia. It is essential to observe the animal eating to decide on the diagnostic steps to follow. Dogs may present with aspiration pneumonia and be critically ill. The dogs should be observed eating to differentiate the different phases of deglutition. Disorders of the oral phase show as inability to masticate and form a bolus, the food will accumulate in the vestibule pouch or fall from the mouth. Disorders of the oesophageal phase present as an animal that forms a bolus and swallows the entire bolus but then regurgitates; these animals tend to have no problem eating and forming the bolus. The disorder lies with primary and secondary peristalsis of the oesophagus in transporting food from the pharynx to the cardia ${ }^{16,19}$. The pharyngeal phase will present as a dog making repeated attempts to swallow a well formed bolus with retching, gagging and possible aspiration or regurgitation of the bolus. These animals will extend the neck and constantly swallow. Some of the bolus may eventually pass through into the oesophagus. The oesophageal motility is normal and the bolus passes into the stomach. These manifestations can be difficult to evaluate in real-time motion and cannot be used to make a diagnosis. They are, however, suggestive of a clinical disease of the pharyngeal phase. There is no diagnostic or specific clinical sign that will confirm a diagnosis, but the clinical signs will indicate a disease of the swallowing process $^{16,19}$.

Oral examination is imperative to rule out oral causes of dysphagia. Oral trauma and dental disease may be obvious on routine clinical examination. Palpation of the cervical area can help to detect a mass but a full oral examination has to be made in all cases even if the cause appears obvious. This will require sedation in most animals and should only be done after 
thoracic radiographs to rule out aspiration pneumonia have been taken. A secondary cleft palate will be obvious on oral examination. Clefts of the soft palate may be missed if they present as a lateral or central cleft. It is important to observe the nasopharynx by rostral retraction of the soft palate in all animals undergoing an oral examination to rule out a nasopharyngeal foreign body/mass and nasal dysgenesis of the dachshund ${ }^{4,5}$.

Electromyography may be useful in ruling out denervation leading to dysphagia but may be completely normal. Its use has not been studied completely and it is not often used. The results of EMG must be interpreted with caution for determining the location of a dysphagia ${ }^{13,16,19}$. The best method to appreciate pathology leading to diffuse denervation of the oropharynx would be a full oral examination under light sedation. This allows the clinician to elicit a gag reflex and to observe the normal symmetrical motion of the arytenoid cartilages."

\section{Clinical pathology}

Laboratory findings vary depending on the animal's condition. Most of the patients have normal laboratory findings. There are no pathogonomic laboratory findings for CA. Patients that show signs of systemic disease should have a full haematology, and chemistry screen and urinalysis should be done ${ }^{4,7}$. The results of these vary depending on the underlying systemic condition and will be as the result of aspiration, malnutrition or underlying disease. Hypothyroidism should be ruled out as a cause of the dysphagia prior to surgical treatment of CA. This should be evaluated in light of the haematology, serum biochemistry and total T4 and TSH. Acetylcholine receptor antibodies are negative and myasthenia gravis should be ruled out clinically and with blood analysis ${ }^{7}$. If these underlying causes are not ruled out the results of corrective surgery for CA will be devastating.

Analysis of the cerebro-spinal fluid is indicated if there are clinical signs of central nervous system dysfunction. In the case of inflammatory and infectious disease it would be unusual for the caudal cranial nerves to be affected without any other clinical signs. An MRI scan is indicated if neurological disease is suspected to detect anatomical changes in the brain such as hydrocephalus ${ }^{7}$.

\section{Radiology}

Thoracic radiographs should form a basis of the diagnostic database. They will rule out diseases of the oesophagus, vascular ring anomalies and hiatal her- nias $^{4,7}$. Thoracic radiographs will help to evaluate the patient for secondary aspiration pneumonia. The gold standard for diagnosis is a barium swallowing study using videofluoroscopy ${ }^{13,16,18,19}$. This gives a real-time image of the phases of deglutition. Barium is the contrast medium of choice but aspiration of the barium during the fluoroscopy is a reported complication $^{16,18,19}$. The barium paste seems to cause more ill effects when aspirated compared with a gruel mixed with food and seems to be more difficult for the animal to expel from the upper airways. It may be an option to use soluble isotonic iodine solutions. However, how the iodine solutions would mix with a gruel and hence the image quality that would be attained during the study are unknown. No ill effects have been seen in 2 clinical cases done by the author up to 24 months after aspiration during fluoroscopy. However, the presence of the foreign material that is very slowly removed via macrophages will worsen any aspiration pneumonia present. It has been reported in humans that barium sulphate can cause a non-fibrotic form of pneumoconiosis ${ }^{2,6}$.

Placing the animal in right lateral recumbency under restraint is the described positioning for a barium swallow. This can affect the swallowing of compromised patients, raising the problem of the radiation safety of the handlers as sedating the patients will affect the study $^{16,18,19}$. It is ideal to use a horizontal beam technique and place the animal in an upright perspex crate restricting its movement. It is best to record the videofluoroscopy study as the phases are rapid. This allows evaluation of the real-time images later in a quiet area without having to worry about the positioning and restraint of the animal. It will allow the images to be slowed down and the phases evaluated in detail ${ }^{16,18,19}$. Studies in normal dogs have shown that restraint in lateral recumbency and light sedation do not affect the normal swallowing phases but this has not been investigated in dogs with a clinical condition and should if possible be avoided as a variable in making the diagnosis ${ }^{19}$.

During deglutition in a case of CA the videofluoroscopy study will show normal oral and pharyngeal transport of the barium bolus to the UES. There will be an inappropriate or non-opening of the UES with retention of the most of the bolus in the pharynx ${ }^{16,18,19}$. This can induce repeated ineffectual attempts at swallowing with consequent aspiration or regurgitation. This is in contrast to pharyngeal dysphagia, where the oral phase is normal but there is inadequate pharyngeal peristalsis leading to retention of much of the bolus in the pharynx. There may be aspiration, regurgitation or reflux into the nasopharyn $x^{16,18,19}$. Post-videofluoroscopy there may be small to moderate amounts of medium retained in the pharynx while a small amount or none passes into the stomach via the oesophagus. There should be minimal to no contrast medium retained in the oesophagus, indicating a normal oesophageal phase of swallow$\operatorname{ing}^{13,18,19}$.

The diagnosis is made on visualisation of the typical signs of asynchronous muscular contraction of the UES in the presence of a strong to normal pharyngeal phase of deglutition. A statistically significant delay in the time between the onset of swallowing and the opening of the UES has been shown to coincide with dogs diagnosed with $C^{13}$. This may help in making a pre-surgical diagnosis.

Pressure manometry during the videofluoroscopy is the ideal but is technically demanding and may add extra time and procedural difficulties to the study and is not commonly available. It is therefore not often used ${ }^{13,16,18,19}$.

If there is doubt as to where the achalasia occurs, then a lateral surgical approach is made to the pharynx. The cricopharyngeal and thyopharyngeal muscles are identified. Metal clips are placed on the cranial and caudal borders of the muscles. Fluoroscopy can then be repeated. The clips will allow the radiologist to identify which muscle is involved as the borders are now highlighted by the radio-opaque $\operatorname{clips}^{4,8}$. This can also be used post-surgery to evaluate the success of the myotomy/myectomy with clips being placed during the surgery ${ }^{4,8}$.

\section{Endoscopy}

Visualisation of the pharynx and the buccal cavity is normal ${ }^{4,7,9}$. No clinical abnormalities should be seen. It has been reported that the pharyngeal recesses, mainly the piriform and vallecular recesses seem to be enlarged ${ }^{16,19}$. However, it is required to rule out many of the differential diagnoses. A complete oral, pharyngeal and laryngeal examination can be made under sedation. It is important to observe the movement of the arytenoid cartilages of the larynx in the absence of EMG. If the area is denervated, movement of these cartilages may be decreased or absent ${ }^{1,13,19}$. Myectomy/myotomy is contraindicated. The soft palate should be evaluated for a secondary cleft palate. Endoscopy of the oesophagus and trachea requires general anaesthesia. In cases of aspiration pneumonia it allows a bronchoalveolar lavage to be performed and samples taken for bacterial culture 
and antibiogram ${ }^{4,8,9}$. Oesophagoscopy should be performed to evaluate the oesophagus for any abnormalities such as stricture, oesophagitis, spirocercosis and other diseases ${ }^{7}$. Spirocercosis tends to be a disease of older animals, with the youngest reported case in a 7-month-old dog. This is due to the relatively long prepatent period of the parasite.

\section{TREATMENT}

\section{Conservative}

Conservative treatment consists of mainly symptomatic treatment to prepare the patient for surgery. This will vary from patient to patient, some patients will tolerate soft food and others hard foods ${ }^{4,5,9,11}$. No specific consistency of food has shown to be better tolerated, and no specific alteration in position of the animal during feeding has been shown to improve the clinical outcome. It is important to ensure that the patient is well hydrated and nourished. If the animal cannot get food per os due to a severe dysfunction, a percutaneous endoscopic gastrostomy (PEG) tube can be placed to feed the patient prior to surgery ${ }^{4,7}$.

Botulism toxin type A has been suggested to be helpful in humans with upper oesophageal sphincter dysfunction. Botulism toxin is injected into the cricopharyngeal muscle under electromyographic guidance, causing a temporary paralysis of the muscle and a resolution of the achalasia in humans ${ }^{6,9,10}$. Endoscopic guidance can be used to aid injection. This can be used in patients with severe aspiration pneumonia that needs intensive treatment to stabilise them prior to surgery, in patients that are undernourished in which a PEG tube is contraindicated or considered more invasive, or in patients that are considered to be too young and the risk of surgery is therefore considered too great ${ }^{6,10}$. The paralysis will last up to 6 months and has been very effective in human paediatrics as a method to alleviate clinical signs temporarily prior to definitive surgical correction or as a therapeutic option ${ }^{6,10}$. This could have clinical applications prior to surgery in the compromised canine patient.

\section{Surgical treatment}

Curative surgical treatment of choice is a cricopharyngeal myectomy. It is critical that an accurate diagnosis is made as a cricopharyngeal myectomy performed in patients with a pharyngeal dysphagia will lead to worsening of the dysphagia and aspiration pneumonia ${ }^{4,5,7,9,16}$. Previous reports show a very poor outcome in surgical treatment in patients with under- lying disease leading to poor pharyngeal function and these patients should not have surgical correction preformed ${ }^{1,13,18,19}$. The procedure should be performed unilaterally as the muscle forms a band making up the upper oesophageal sphincter. By resecting the band the asynchronous contraction is stopped, thus relieving the dysphagia. Some surgeons advocate removal of a $1 \mathrm{~cm}$ portion of the cricopharyngeal muscle ${ }^{4}$. This is thought to prevent adhesion formation between the resected ends and re-formation of the band by fibrous tissue joining up the ends of the resected muscle. Others simply perform a myotomy $y^{1,4,11,14,15}$. Other surgical options have been reported in humans such as pneumatic dilation, bouginage and neurectomy of the pharyngeal plexus. Evidence is, however, lacking as to their affects in resolving clinical disease and they pose several risks ${ }^{2,6,10}$.

\section{Positioning}

The patient is positioned in dorsal recumbency with the legs pulled caudally. A sandbag or $1 \ell$ drip bag can be placed under the neck to elevate the surgical area. The hair should be liberally clipped from $1 \mathrm{~cm}$ cranial to the angle of the mandible to the level of the thoracic inlet $^{4,8,9,11}$.

\section{Surgery}

The approach is via a ventral midline cervical incision from just cranial to the larynx to the mid-cervical region. The paired sternohyoidus muscles must be identified and separated in the midline without cutting through any of the fibres.The larynx and trachea should be gently retracted and rotated laterally using traction on the sternothyroidus muscle to expose the cricopharyngeal musculature. This retraction can be maintained by a stay suture placed through the lamina of the thyroid cartilage of the larynx At this point it is very helpful for an assistant not surgically sterile to pass an orogastric tube into the oesophagus to aid identification. The cricopharyngeal muscle should be identified and incised on its midline. Ensure that all fibres of the muscle on the oesophagus are elevated and incised ${ }^{4,8,11}$. The thyropharyngeus muscle can be incised to ensure complete transection and alleviation of the achalasia. Complete division of the fibres of the cricopharyngeal muscle is essential to a successful postoperative outcome. Iatrogenic damage and perforation of the oesophageal wall must be avoided. A myectomy may be performed as above but then followed a parallel incision and removing a strip of the cricopharyngeal muscle about 0.5 to $1 \mathrm{~cm}$ wide. Vigorous lavage of the surgical site must be performed $^{4,8,15}$. Appose the sternohyoidus muscles with a simple continuous pattern. Closure of the subcutaneous tissues and skin is routine.

\section{Postoperative care}

It is recommended to feed the dogs soft food or gruel for 1-2 days post-surgery and slowly return them to normal food over the next 2-3 weeks. This will vary according to the clinical status of the dog at the time of surgery. Once the surgery has been completed there is resolution of the achalasia and dogs will be able to tolerate varying consistencies of food ${ }^{1,5,9}$. Owing to pain and swelling soft food is preferred in the immediate postoperative period. If there is still a visible dysphagia after 2-3 days it should be suspected that an incomplete myectomy or myotomy was performed or the diagnosis was incorrect. The achalasia should be resolved as soon as a complete myectomy/myotomy has been performed and often no special care is needed ${ }^{4,8,9}$. Antibiotics are not needed in the postoperative period as they show little benefit in preventing wound infections. This varies according to the status of the patient and the presence of aspiration pneumonia. In humans the link between CA and gastrooesophageal reflux (GER) has been found $^{6}$. It is not clear if the GER leads to hyper-reactivity of the cricopharyngeal muscle resulting in achalasia and consequent spasm. However, it is recommended that after cricopharyngeal myectomy GER needs to be addressed in humans. Following the loss of the cricopharyngeal muscle and hence the upper oesophageal sphincter GER may be increased $^{6}$. The recommended treatment reported in humans is the use of prokinetic agents such as metoclopramide combined with decreasing gastric acidity with $\mathrm{H}-2$ blockers. Sucralfate can be used in treating any reflux oesophagitis.

\section{Complications}

The reported complications include fibrosis with return of achalasia, recurrent laryngeal paralysis, oesophageal perforation, pharyngocutaneous fistula and infection. The biggest potential complication is if there is an underlying condition, for example a neuromuscular disease, when the surgery will worsen the clinical signs and induce aspiration pneumonia $^{4,8,9}$. This is due to reflux of the bolus through a non-existent UES as the underlying disease will affect pharyngeal muscle function and oesophageal muscle function $^{7,18,19}$. 


\section{PROGNOSIS}

If the myectomy has been performed successfully then the prognosis is excellent for full recovery and quality of life. The true achalasia with no underlying disease or concurrent oesophageal hypomotility carries an excellent prognosis ${ }^{4,7,9}$. However, aspiration pneumonia that cannot be controlled will lead to a poor outcome. If the diagnostic steps are not followed and the myectomy is performed for a pharyngeal dysphagia other than cricopharyngeal achalasia, the results will be worsening of the aspiration pneumonia. If there is underlying megaoesophagus, the dog will deteriorate owing to worsening of the aspiration pneumonia as food will now reflux through a non-existent $\mathrm{UES}^{7}$. It appears that young animals with the true form of congenital CA with no underlying neuromuscular, inflammatory, anatomic or functional abnormalities carry a good long-term prognosis $^{4,7,9,11}$. This correlates with the clinical outcome achieved in the 2 patients treated with a myectomy by the author.

\section{REFERENCES}

1. Allen S W 1991 Surgical management of pharyngeal disorders in the dog \& cat. Problems in Veterinary Medicine 3: 290-297

2. Davis M V, Merati A L, Safwan S, Jaradeh S,
Blumin J H 2007 Myosin heavy chain composition and fibre size of the cricopharyngeus muscle in patients with achalasia and normal subjects. Annals of Otology, Rhinology and Laryngology 116: 643-646

3. Evans H E 1993 Millers anatomy of the dog (3rd edn). W B Saunders Company, Philadelphia

4. Fossum T W, Hedlund C S 2007 Surgery of the digestive system. In Fossum $\mathrm{T} W$, Hedlund C S, Hulse D A, Johnson A L, Seim H B, Willard M D, Carroll G L (eds) Small animal surgery Vol. 3. Mosby, St Louis, Missouri: 339-530

5. Goring R L, Kagan K G 1982 Cricopharyngeal achalasia in the dog: radiographic evaluation and surgical management. Compendium on Continuing Education for the Practicing Veterinarian 4: 438-444

6. Jain V, Bhatnagar V 2009 Cricopharyngeal myotomy for the treatment of cricopharyngeal achalasia. Journal of Pediatric Surgery 44: 1656-1658

7. Jergens A E 2000 Diseases of the oesophagus. In Ettinger S J, Feldman E C (eds) Textbook of veterinary internal medicine Vol. 2. Elsevier Saunders, St Louis, Missouri: 1487-1499

8. Kyles A E 2003 Oesophagus. In Slatter D (ed.) Textbook of small animal surgery Vol. 3. Saunders, Philadelphia: 573-592

9. Ladlow J, Hardie R J 2000 Cricopharyngeal achalasia in dogs. Compendium on Continuing Education for the Practicing Veterinarian 22: 750-755

10. Lee S Y, Seo H G, Paik N J 2009 Botulinum toxin injection for dysphagia. American Journal of Physical Medicine $\mathcal{E}$ Rehabilitation 88: $491-494$
11. Niles J D, Williams J M, Sullivan M, Crowsley F E 2001 Resolution of dysphagia following cricopharyngeal myectomy in six young dogs. Journal of Small Animal Practice 42: 32-35

12. Pfeifer R M 2003 Cricopharyngeal achalasia in a dog. Canadian Veterinary Journal 44: 993-995

13. Pollard R E, Marks S L, Davidson A, Hornof W J 2000 Quantitative videofluoroscopic evaluation of the pharyngeal function in the dog. Veterinary Radiology \& Ultrasound 41: 409-412

14. Rosin E, Hanlon G F 1972 Canine cricopharyngeal achalasia. Journal of the American Veterinary Medical Association 160: 14961499

15. Sokolovsky V 1967 Cricopharyngeal achalasia in a dog. Journal of the American Veterinary Medical Association 150: 281-285

16. Suter P F, Watrous B J 1980 Oropharyngeal dysphagias in the dog: a cinefluorographic analysis of experimentally induced and spontaneously occurring swallowing disorders. Veterinary Radiology 21: 24-30

17. Warnock J J, Marks S L, Pollard R, Kyles A E, Davidson A 2003 Surgical management of cricopharyngeal dysphagia in dogs: 14 cases (1989-2001). Journal of the American Veterinary Medical Association 223: 1462-1468

18. Watrous B J, 2007 Oesophagus. In Thrall D E (ed.) Textbook of veterinary diagnostic radiology, Vol. 5. Saunders Elsevier, St Louis, Missouri: 495-511

19. Watrous B J, Suter P F 1979 Normal swallowing in the dog: A cineradiographic study. Veterinary Radiology 20: 99-109 